\title{
SUPPORT PROJECTIONS ON BANACH SPACES
}

\author{
by P. G. SPAIN
}

(Received 21 May, 1975)

Each bounded linear operator $a$ on a Hilbert space $K$ has a hermitian left-support projection $p$ such that $p K=\overline{a K}=\overline{a a^{*} K}$ and $(1-p) K=\operatorname{ker} a^{*}=\operatorname{ker} a a^{*}$. I demonstrate here that certain operators on Banach spaces also have left supports.

Throughout this paper $X$ will be a complex Banach space with norm-dual $X^{\prime}$, and $L(X)$ will be the Banach algebra of bounded linear operators on $X$. Two linear subspaces $Y$ and $Z$ of $X$ are orthogonal (in the sense of G. Birkhoff) if $\|y\| \leqq\|y+z\|(y \in Y, z \in Z)$; this orthogonality relation is not, in general, symmetric. It is easy to see that $p X$ is orthogonal to $(1-p) X$ if and only if the norm of $p$ is 0 or 1 , when $p$ is a projection on $X$.

An element $h$ of a complex unital Banach algebra $A$ is hermitian if $\| \exp ($ ith $) \|=1(t \in \mathbf{R})$; equivalently, $h$ is hermitian if its numerical range, $\left\{f(h): f \in A^{\prime}, f(1)=\|f\|=1\right\}$, is real.

Proposition. Let $X$ be a reflexive Banach space and let $h$ be a hermitian operator on $X$ (that is, a hermitian element of $L(X)$ ). Then there is a projection $p$ of norm 0 or 1 such that

$$
p X=\operatorname{ker} h \text { and }(1-p) X=\overline{h X} ;
$$

so $\operatorname{ker} h$ is orthogonal to $h X$.

Proof. This proposition is an immediate consequence of $[4:$ VII. 7.5$]$ and the inequality $\left\|\alpha(\alpha-h)^{-1}\right\| \leqq 1$ which holds for hermitian $h$ and purely imaginary $\alpha$. To prove this inequality, put $k=(\alpha-h)^{-1}$ and choose $f$ in $A^{\prime}$ with $\|f\|=1, f(k)=\|k\|$; define $g$ in $A^{\prime}$ by $g(a)=\|k\|^{-1} f(a k)$; then $g(1)=\|g\|=1$; so, $g(h)$ being real, $|\alpha| \leqq|\alpha-g(h)|=$ $|g(\alpha-h)|=\|k\|^{-1}|f(1)| \leqq\|k\|^{-1}$.

Alternatively, the result can be derived from [6] where it is shown that, first, ker $t$ is orthogonal to $\overline{t X}$ for any operator $t$ the boundary of whose numerical range contains 0 , and, second, that $X=\overline{t X} \oplus \operatorname{ker} t$ if $X$ is reflexive.

The Vidav-Palmer theorem $[2, \S 6]$ characterises unital $C^{*}$-algebras among unital Banach algebras; a unital Banach algebra $A$ is a $C^{*}$-algebra if and only if $A=H+i H$, where $H$ is the set of hermitian elements of $A$. I say that $A$ is a $V^{*}$-algebra (on $X$ ) if $A$ contains the identity operator on $X$ and $A=H+i H$ (so that $A$ is, abstractly, a $C^{*}$-algebra).

Suppose that $A$ is a $V^{*}$-algebra on $X$ and that its closed unit ball $A_{1}$ is relatively compact in the weak operator topology; this will happen if $X$ is reflexive [8] or if $X$ is weakly sequentially complete and $A$ is commutative [7, Theorem 2 and Corollary 2]. Let $\tilde{A}$ be the linear span of the closure $\left(A_{1}\right)^{w}$ of $A_{1}$ in the weak operator topology: $\tilde{A}=\cup\left\{k\left(A_{1}\right)^{w}: k \in \mathbf{N}\right\}$. Then $\tilde{A}$ is a $V^{*}$-algebra; indeed, $\tilde{A}$ is a $W^{*}$-algebra (an abstract von Neumann algebra) [8].

I say that an element $n$ of a unital Banach algebra $A$ is normal if $n$ can be expressed as $h+i k$ where $h$ and $k$ commute and $h^{r} k^{s}$ is hermitian $(r, s=0,1,2, \ldots)$; so $n$ is normal if and only if there is a commutative subalgebra of $A$ which contains $n$ and the identity of $A$ 
and, further, is a $C^{*}$-algebra. In particular, $n$ is a normal operator on $X$ (that is, normal in $L(X)$ ) if and only if $n$ belongs to a commutative $V^{*}$-algebra on $X$. (This definition of normality is narrower than that in [2].)

THEOREM. Let $X$ be a complex Banach space, let $A$ be a $V^{*}$-algebra on $X$ with weakly relatively compact unit ball and let $a \in A$. Then a has a hermitian left-support projection $p$ such that

$$
p X=\overline{a X}=\overline{a a^{*} X} \text { and }(1-p) X=\operatorname{ker} a^{*}=\operatorname{ker} a a^{*} .
$$

Moreover, $p X$ and $(1-p) X$ are mutually orthogonal. Further, $\operatorname{ker} a=\operatorname{ker} a^{*}$ if $a$ is normal.

Proof. Let $B$ be the subalgebra generated by $a a^{*}$. Write $C$ for the closure of $B$ in the topology $\sigma$ induced on $A$ by its predual. Let $p$ be the identity of the $W^{*}$-algebra $C$. By Kaplansky's density theorem [5, 1.9] there exists a bounded net $P$ (in $B$ ) which $\sigma$-converges to $p$. But then $P$ converges to $p$ in the weak operator topology (for on $A_{1}$ these two topologies are comparable, compact and Hausdorff: hence identical). Thus there exists a bounded net $Q$ (of convex combinations of $P$ ) which converges to $p$ in the strong operator topology. So $p X \subseteq \overline{a a^{*} X} \subseteq \overline{a X}$ and $(1-p) X \subseteq \operatorname{ker} a^{*}$. Now $0=(1-p)\left(a a^{*}\right)=(a-p a)(a-p a)^{*}$; so $a=p a$; from which $\overline{a a^{*} X} \subseteq \overline{a X} \subseteq p X$ and ker $a a^{*} \subseteq(1-p) X$. Therefore $p X=\overline{a X}=\overline{a a^{*} X}$ and $(1-p) X=\operatorname{ker} a^{*}=\operatorname{ker} a a^{*}$. The norms of $p$ and $1-p$ are 0 or 1 , because $p$ is hermitian; so $p X$ and $(1-p) X$ are mutually orthogonal. Finally, if $a$ is normal, then

$$
\operatorname{ker} a=\operatorname{ker} a^{*} a=\operatorname{ker} a a^{*}=\operatorname{ker} a^{*} .
$$

This theorem generalises Lemma 3.1 of [1].

COROLlary. Let $s$ be a scalar-type spectral operator on a Banach space $X$. Then $X=\overrightarrow{s X} \oplus \operatorname{ker} s$.

Proof. There is a spectral measure $e$ supported by the spectrum of $s$ with $s=\int z e(d z)$ $[4, \mathrm{XV}]$. Now $X$ can be given a new norm (equivalent to the original norm) with respect to which all the values of $e$ are hermitian. (This is a result of E. Berkson: see [3, §33].) Thus $s$ is normal (for the new norm). Also, by Theorem 2 of [7], the norm-closed algebra generated by $e$ has weakly relatively compact unit ball. The theorem may therefore be applied to give $\operatorname{ker} s=\operatorname{ker} s^{*}$ and $X=\bar{s} X \oplus \operatorname{ker} s$.

H. R. Dowson has remarked to me that this corollary can be derived from results of S. R. Foguel; see [4, XV. 8.2 and 8.3].

The corollary extends neither to spectral operators in general (consider $\left[\begin{array}{ll}0 & 1 \\ 0 & 0\end{array}\right]$ acting on $\mathbf{C}^{2}$ ) nor to all scalar-type prespectral operators (the operator $s$ on $l^{\infty}$ defined by $s\left(x_{n}\right)=\left(n^{-1} x_{n}\right)$ has zero kernel and separable range).

The proposition and theorem suggest the question: must $X=\overrightarrow{h X} \oplus \operatorname{ker} h$ whenever $X$ is weakly complete and $h$ is a hermitian operator on $X$ ? 


\section{REFERENCES} 7-18.

1. B. A. Barnes, Representations of $B^{*}$-algebras on Banach spaces, Pacific J. Math. 50 (1974),

2. F. F. Bonsall and J. Duncan, Numerical ranges of operators on normed spaces and of elements of normed algebras (Cambridge U.P., 1971).

3. F. F. Bonsall and J. Duncan, Numerical ranges II (Cambridge U.P., 1973).

4. N. Dunford and J. T. Schwartz, Linear operators (Interscience, 1958, 1963, 1971).

5. S. Sakai, $C^{*}$-algebras and $W^{*}$-algebras (Springer-Verlag, 1971).

6. A. M. Sinclair, Eigenvalues in the boundary of the numerical range, Pacific J. Math. 35 (1970), 231-234.

7. P. G. Spain, On commutative $V^{*}$-algebras II, Glasgow Math. J. 13 (1972), 129-134.

8. P. G. Spain, The $W^{*}$-closure of a $V^{*}$-algebra, J. London Math. Soc. (2) 7 (1973), 385-386.

Department of Mathematics

UNIVERSITY OF GLASGOW 